\title{
Enhanced recellularization of renal extracellular matrix scaffold under negative pressure
}

\author{
Satoshi Hachisuka, Yuichi Sato*, Miki Yoshiike, Ryuto Nakazawa, Hideo Sasaki, and Tatsuya Chikaraishi \\ Department of Urology, St. Marianna University School of Medicine, Kawasaki 216-8511, Japan
}

\begin{abstract}
To overcome the scarcity of organs available for clinical transplantation, whole-organ engineering has been attempted in recent years. In this approach, cellular components of an organ are totally removed through arterial perfusion with a detergent, and the resulting extracellular matrix scaffold serves as a template for subsequent cell seeding to create a new organ. However, the low efficiency of recellularization impedes generation of a fully functioning organ by this method. To improve the efficiency of recellularization, we decellularized murine kidneys and subsequently performed recellularization of the kidney scaffolds in a negativepressure environment. A pressure gradient was applied by placing the scaffolds in a custom-build device that could remove air to create a vacuum. Recellularization was done antegradely via the arterial route or retrogradely via the ureteral route. After endothelial cells were seeded intra-arterially or tubular cells were instilled into the ureter, we counted the cells in cross sections through the renal hilum. Cells were successfully seeded without damage to the scaffold. By applying different pressure gradients that were determined in an empirical fashion, we observed an increase of engrafted cells in both the vessels and the parenchyma, although seeding of the parenchyma seemed to be less efficient. Seeded endothelial cells survived and proliferated during 72-hour perfusion culture, and vascular resistance increased along with increased cellularity of the kidney scaffolds. In conclusion, the present seeding method employing a pressure gradient is safe and effective. The strength of the gradient applied should differ between the arterial and ureteral seeding routes. This method may be useful to improve seeding efficiency and assist progress towards the ultimate goal of developing a functioning organ for clinical use.
\end{abstract}

\section{Introduction}

Organ transplantation has been performed to treat end-stage disease of various organs with substantial success, but there is a limited supply of donor organs that makes it impossible to treat every patient in need of transplantation. In Japan for instance, about 12,000 patients are currently waiting for kidney transplantation and more than 300,000 individuals are on hemodialysis with end-stage kidney disease [1]. However, only some 1500 patients undergo kidney transplantation every year [2].

To deal with the scarcity of organs for transplantation, a novel method of creating new organs has been investigated in recent years [3-5]. In this new approach, the cellular components of a whole organ are totally removed through arterial perfusion using a detergent, and the resulting extracellular matrix (ECM) scaffold is employed as a biological template for cell seeding. The ultimate goal is to achieve $e x$ vivo generation of a functioning organ.

This method has been applied for various organs, including the heart, liver, kidneys, lungs, and others [6-13], but creation of a fully functioning organ has not yet been achieved. Although there are a number of hurdles to overcome, low efficiency of recellularization is one of the crucial factors that need to be addressed. Therefore, the present study was performed to investigate the improvement of recellularization efficiency. After we decellularized whole murine kidneys, recellularization of the kidney scaffolds was subsequently done under a negative-pressure environment, and we examined whether this facilitated the recellularization process. In addition, we perfused and cultured recellularized scaffolds to determine whether recellularization allowed the development of organ-specific functions.

\section{Materials and methods}

\section{Kidney decellularization}

We used adult male Sprague-Dawley rats (Clea Japan, Inc., Tokyo, Japan) weighing 300-500 g in accordance with our institutional regulations. After anesthesia was induced by intraperitoneal injection of pentobarbital $(0.05 \mathrm{mg} / \mathrm{g})$ and systemic heparinization $(0.4 \mathrm{iu} / \mathrm{g})$ was done via the femoral vein, the left kidney was exposed by midline laparotomy. The aorta was perfused with normal saline to remove blood, and then the left renal artery was cannulated with a 24-gauge cannula (Becton, Dickinson and Company) to allow perfusion. We also inserted the same type of cannula into the ureter.

We isolated the kidney and performed decellularization by a modification of the method of Uygun et al. [7]. In brief, after perfusing the renal artery with normal saline at $50 \mathrm{~cm} \mathrm{H}_{2} \mathrm{O}$ for 30 minutes to remove residual blood, we infused increasing concentrations of sodium dodecyl sulfate (SDS) at $1 \mathrm{ml} / \mathrm{min}$ in the following order: $0.01 \%$ for 12 hours, $0.1 \%$ for 12 hours, $1 \%$ for 24 hours. SDS was delivered via a peristaltic pump (Perista ${ }^{\mathrm{TM}}$ Pump, Atto Corporation, Tokyo, Japan). After decellularization, we washed the resulting kidney scaffold with phosphate-buffered saline (PBS) for 30 minutes and with $1 \%$ Triton X-100 for 30 minutes, and performed a final wash with PBS containing

Correspondence to: Yuichi Sato MD, Ph.D, Department of Urology, St. Marianna University School of Medicine, 2-16-1 Sugao, Miyamae, Kawasaki 216-8511, Japan, Tel: +81-44-977-8111; Fax: +81-44-977-0415; E-mail: sato@marianna-u.ac.jp

Received: October 17, 2015; Accepted: October 27, 2015; Published: October 31,2015 
penicillin-streptomycin and $0.05 \%$ sodium azide for 60 minutes. We then stored the scaffolds at $-4^{\circ} \mathrm{C}$.

\section{Characterization of decellularized kidney scaffolds}

For histological analysis, decellularized scaffolds were fixed in formalin and sections were cut and stained with hematoxylin and eosin (HE). Scaffolds fixed in $2.5 \%$ glutaraldehyde were also analyzed by scanning electron microscopy (SEM). Immunohistochemistry for type IV collagen, laminin was performed to evaluate the retention of these basement membrane proteins by using anti-type IV collagen antibody (Southern Biotech, Birmingham, USA), anti-laminin antibody $\left(\mathrm{Abcam}^{\mathrm{TM}}\right.$, Cambridge, UK). Nuclei were counterstained with 4',6-diamidino-2-phenylindole (DAPI, ThermoFisher Scientific, Massachusetts, USA).

\section{Scaffold recellularization via the antegrade and retrograde routes}

We seeded the decellularized kidney scaffolds via two routes, which were infusion of rat aortic endothelial (RAE) cells into the renal artery (antegrade seeding) and infusion of rat epithelial tubular cells (NRK52E) into the ureter (retrograde seeding). RAE cells were purchased from VEC Technologies, Inc. (New York, USA) and NRK-52E was purchased from the European Collection of Cell Cultures. RAE cells were suspended at $10 \times 10^{6}$ cells $/ \mathrm{ml}$ in Dulbecco's modified Eagle's medium (DMEM, Gibco ${ }^{\mathrm{TM}}$ ), supplemented with $10 \%$ fetal bovine serum, $1 \%$ non-essential amino acids, and $1 \%$ antibiotic-antimycotic, while NRK-52E were suspended at the same concentration in CS-C complete medium (Cell Systems Corporation).

Then $20 \times 10^{6} \mathrm{RAE}$ cells suspended in $2 \mathrm{ml}$ of medium were manually injected into the renal artery. To prevent sudden elevation of the intra-scaffold pressure, the cells were infused at the rate of $0.4 \mathrm{ml} /$ min or less. To enhance cell dispersion inside the scaffold, we applied a transrenal pressure gradient (TPG) by using a custom-built device that could reduce the air pressure, with the internal pressure being adjusted to $-100 \mathrm{mmHg}$ for $60 \mathrm{~min}$ (Figure 1). We set the device at $-100 \mathrm{mmHg}$

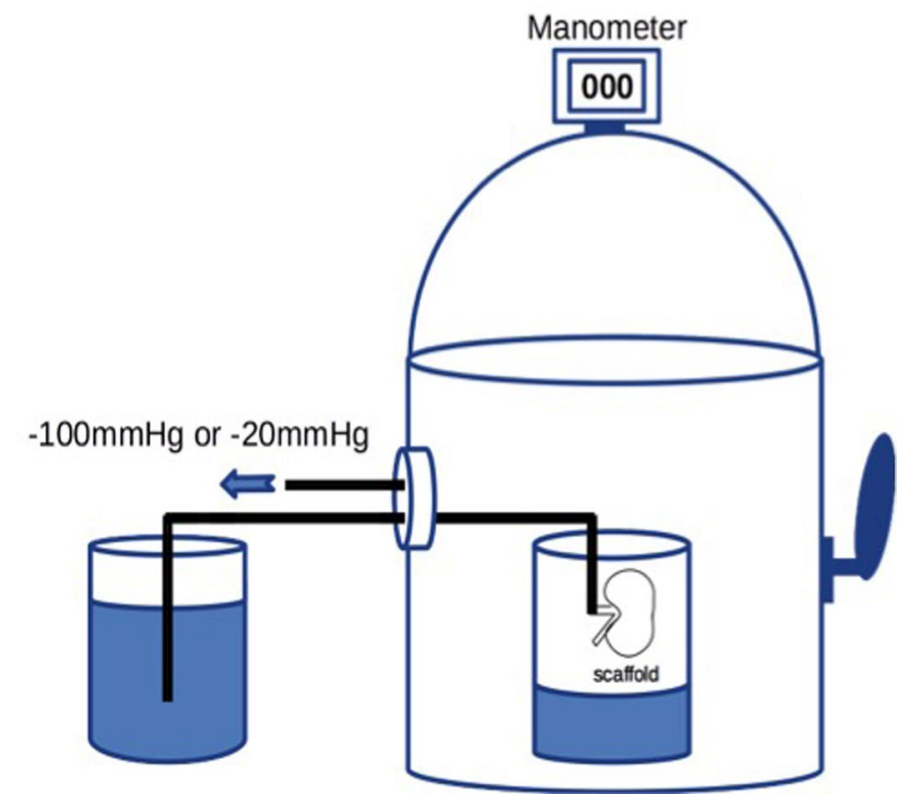

Figure 1. A schematic diagram of the device capable of withdrawing air to create a negative pressure environment. Internal pressure of the chamber can be accurately adjusted by controlling air inflow through manipulating the external lever. because our preliminary study showed that a greater TPG was likely to cause injury to the scaffold. The distal end of the arterial cannula was placed in PBS outside the device to allow free flow of fluid, whereas the ureteral cannula was left inside the device.

For retrograde seeding, we manually infused $1 \times 10^{6}$ RET cells suspended in $0.1 \mathrm{ml}$ of medium into the ureter over one minute. We infused a larger volume of cell suspension in early experiments, but this caused overexpansion and rupture of the renal pelvic wall, leading to leakage of cells from the scaffold. Therefore, we reduced the volume to $0.1 \mathrm{ml}$. After manual bolus infusion of cells into the ureter, we applied TPG in a similar way to antegrade seeding. The seeded scaffold was placed in the device and the pressure was set at $-20 \mathrm{mmHg}$ for $60 \mathrm{~min}$ (Figure 1), while the distal end of the ureteral cannula was placed in PBS outside the device and the arterial cannula remained inside it.

\section{Cellularity after recellularization}

To evaluate the effect of TPG on cell seeding, we compared cellularity between scaffolds repopulated with manual injection only and those repopulated with manual injection + TPG. Immediately after cell seeding, the repopulated scaffolds were processed for HE staining and cell counting. Then we counted engrafted cells in reconstructed images of cross sections through the renal hilum at $x$ 200 magnification. Engrafted RAE cells were counted after antegrade seeding, whereas NRK-52E was counted after retrograde seeding. After antegrade seeding, we also counted the total number of glomeruli and the number of engrafted glomeruli that contained at least a single cell (cell-positive glomeruli), and then we calculated the percentage of cellpositive glomeruli.

\section{Perfusion culture of kidney scaffolds}

To examine whether infused cells could survive and proliferate in the kidney scaffolds, we performed perfusion culture experiments. After seeding RAE cells with TPG, the repopulated scaffolds were immersed in culture medium for 3-5 hours to allow cell attachment. Then each scaffold was mounted in a bioreactor for culture with continuous perfusion via the renal artery. We built a simple bioreactor consisting of a culture medium reservoir, a Perista ${ }^{\mathrm{TM}}$ Pump, and connecting tubes. This was used to recirculate $100 \mathrm{ml}$ of supplemented DMEM at $1 \mathrm{ml} / \mathrm{min}$ for 72 hour, after which the histology of the perfusion-cultured scaffold was assessed. We exchanged the medium at 48 hours and maintained constant culture conditions by placing the system in an incubator. At the end of 72-hour perfusion culture, we processed the scaffolds for HE staining and immunofluorescence for KI-67 (ThermoFisher Scientific, Massachusetts, USA).

For analysis of organ function, we measured the arterial pressure during perfusion of the renal artery in re-endothelialized scaffolds, assuming that endothelial cells could potentially alter flow dynamics inside the scaffold [14]. We used modified Krebs-Henseleit (KH) solution [9] as a surrogate for blood, delivered it into the renal artery (100 $\mathrm{ml}$ in total, over approximately $60 \mathrm{~min}$ ), and then recorded the arterial pressure to calculate the vascular resistance [arterial pressure $(\mathrm{mmHg}) /$ flow rate $(\mathrm{ml} / \mathrm{min})]$. We also recorded the volume of fluid passively drained via the ureter.

\section{Results}

\section{Assessment of decellularized kidney scaffolds}

During continuous arterial perfusion with SDS, the isolated kidney gradually became whitish, and was transparent after 48 hours (Figure 2A). By 


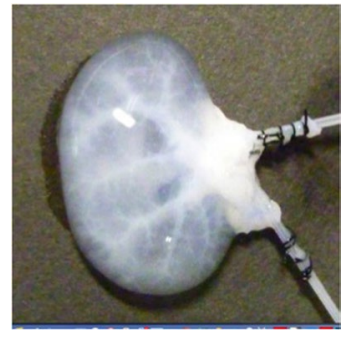

(A)

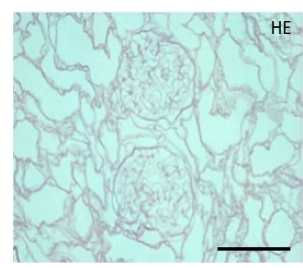

(C)

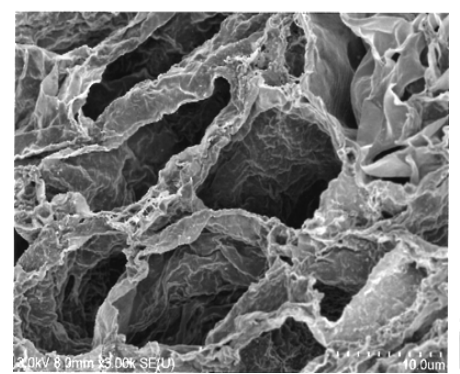

(E)

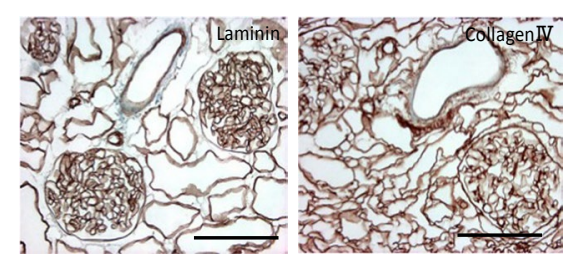

(D)

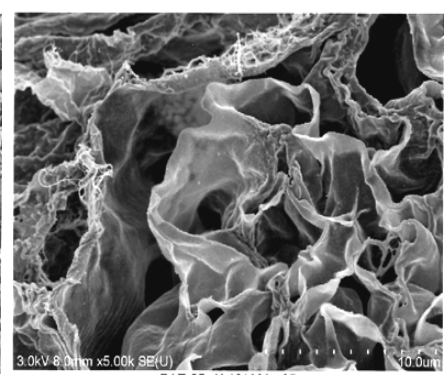

Figure 2. Evaluation of decellularized scaffolds. A: Appearance of decellularized rat kidney after 48-hour continuous perfusion with SDS. The kidney becomes nearly transparent, while retaining its gross appearance.

B: Infusion of dye into the renal artery (left) and the ureter (right). In acellular scaffolds, tree-like vascular network (left) and pelvic spaces (right) are clearly visualized without a major leakage of dye, indicating preservation of innate structures of extracellular matrix

C: A representative HE staining of decellularized rat kidney; cellular and nuclear contents are absent, structures of glomerular and tubular basement membrane are well preserved. Scale bar, $100 \mu \mathrm{m}$

D: Immunohistochemistry for laminin (left) and collagen IV (right); a contiguous network of laminin and collagen IV, primary constituents of renal basement membrane, is displayed in glomerular and tubular compartments. Scale bar, $100 \mu \mathrm{m}$. E: Ultrastructural analysis with scanning electron microscopy; a complete removal of cellular contents and a wellorganized 3 dimensional architecture are shown. Tubular lumen (left) and glomerular capillary lumen (right) are clearly identified.

infusing dye into the renal artery, we showed that the scaffolds retained innate tree-like vasculature. Also, infusion of dye into the ureter confirmed structural retainment of the renal pelvic spaces (Figure 2B). When the decellularized scaffolds were stained with HE, we found that cellular material and nuclei were completely removed while fibrous structures remained (Figure 2C). DAPI staining did not detect cell nuclei in the decellularized scaffolds. Immunofluorescence revealed that type IV collagen and laminin were well preserved in the scaffolds (Figure 2D). SEM showed a reticular structure, in which glomerular and tubular compartments were clearly identified (Figure 2E). Thus, we found that cellular components were adequately removed and innate structures were well preserved in the decellularized scaffolds, which are thought to be prerequisites for successful cell seeding.

\section{Changes of cellularity after seeding}

During antegrade cell seeding, we manually infused $2 \mathrm{ml}$ of cell suspension $\left(20 \times 10^{6}\right.$ RAE cells $)$ into the renal artery without any changes in the appearance of the scaffold. More than $90 \%$ of the infused cells remained inside the scaffold. While TPG was applied, there was about $100 \mathrm{ml}$ of PBS inflow through the arterial cannula. HE staining of the scaffolds repopulated with manual injection only revealed moderate spread of infused cells into the peripheral vasculature and glomeruli (Figure 3A). Meanwhile, after repopulation of scaffolds with manual injection + TPG, there were more cells counted per hilar cross-section, although the change was not statistically significant (Figure 3A). Cellpositive glomeruli showed a significant increase from $72 \%$ to $85 \%$ when TPG was applied (Figure 3B), suggesting that TPG facilitated antegrade dispersion of cells inside the scaffold.

With retrograde seeding, we infused $0.1 \mathrm{ml}$ of cell suspension $(1 \times$ $10^{6} \mathrm{NRK}-52 \mathrm{E}$ ) into the ureter without apparent trauma to the scaffold. Histological assessment revealed that the pelvic wall was intact. NRK$52 \mathrm{E}$ were not dispersed efficiently into the renal parenchyma after manual infusion (Figure 3C). The cells only occasionally reached the tubular spaces and never reached the glomerular spaces. We found layers of viable cells on the renal pelvic wall, suggesting that the wall was a barrier to cell dispersion. When we applied TPG with retrograde cell seeding, there was no inflow of PBS, a phenomenon observed with antegrade seeding. We found a significant increase of engrafted cells

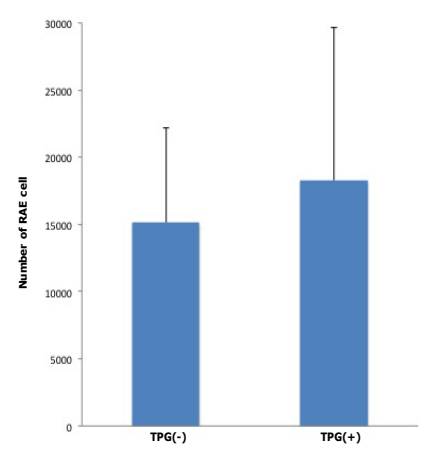

(A)

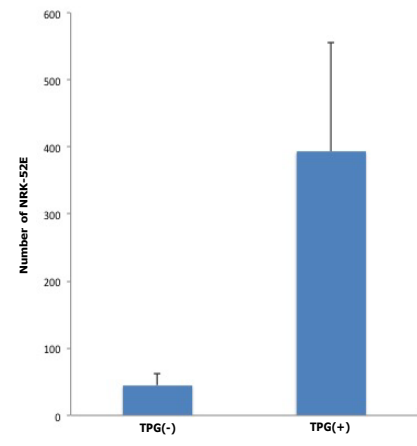

(C)

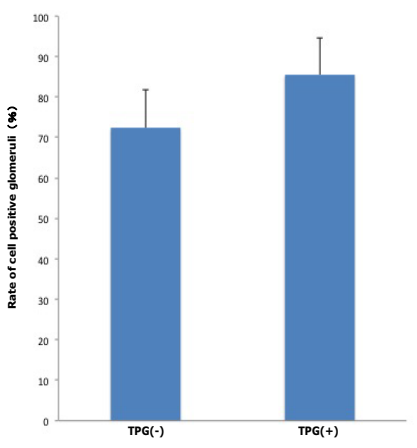

(B)
Figure 3. Changes of cellularity after antegrade $(A, B)$ and retrograde $(C)$ recellularization. A: RAE cell number with and without TPG $(-100 \mathrm{mmHg}$ for $60 \mathrm{~min})$. In cross sectional images through the renal hilum, dispersed endothelial cells (by manual injection + TPG, $\mathrm{n}=5$ ) were greater in number, compared to the control (by manual injection only, $\mathrm{n}=5$ ), although it did not reach a statistical significance ( $\mathrm{p}=0.2$, Student's t-test).

B: Cell positive glomeruli with and without TPG (-100 $\mathrm{mmHg}$ for $60 \mathrm{~min})$. Cell-positive glomeruli (by manual injection $+\mathrm{TPG}, \mathrm{n}=5$ ) were significantly more prevalent, compared to the control (by manual injection only, $\mathrm{n}=5)$. $(\mathrm{p}<0.05)$.

C: Number of NRK-52E with and without TPG (-20 mmHg for $60 \mathrm{~min})$. Dispersed tubular epithelial cells (by manual injection + TPG, $n=5$ ) showed a significant increase $(p<0.05)$, compared to the control (by manual injection only, $\mathrm{n}=5$ ). 
per hilar cross-section (Figure 3C), although the total cell count was still much lower than after antegrade seeding. These results indicate that TPG also facilitated retrograde cell seeding, but to a more limited extent.

\section{Structural and functional analysis of perfusion-cultured kidney scaffolds}

Repopulated kidney scaffolds underwent perfusion culture for 72 hours at the rate of $1 \mathrm{ml} / \mathrm{min}$, with no obvious elevation of pressure that could potentially damage the scaffolds. Histological examination revealed that endothelial cells were retained and survived in the vasculature and glomeruli (Figure 4A). We also found endothelial cells proliferated in the vasculature, as indicated by positive KI-67 staining,

(A)

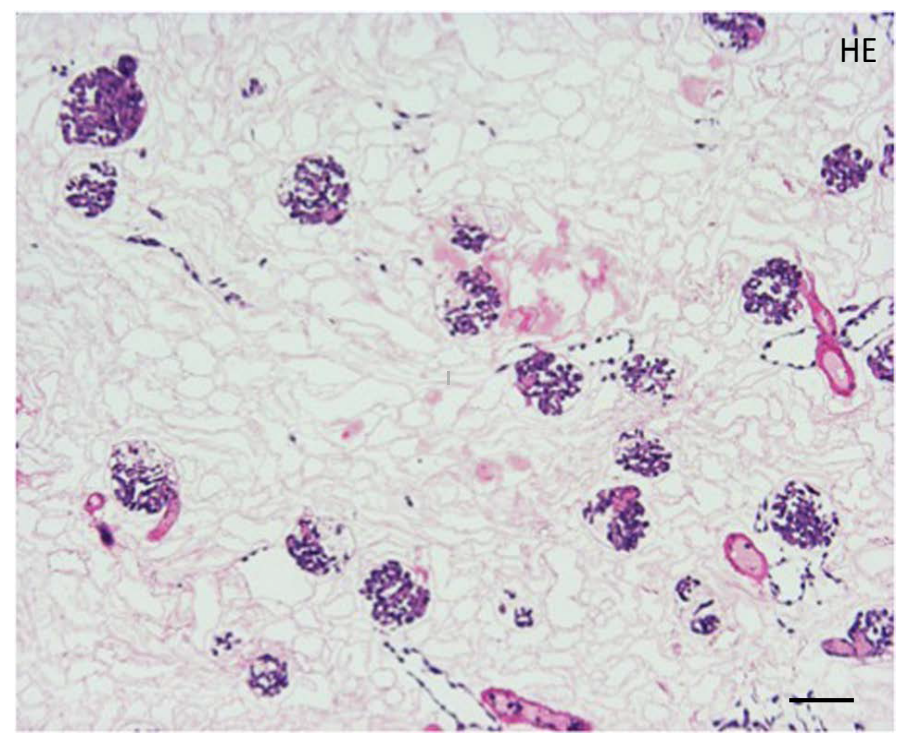

(B)

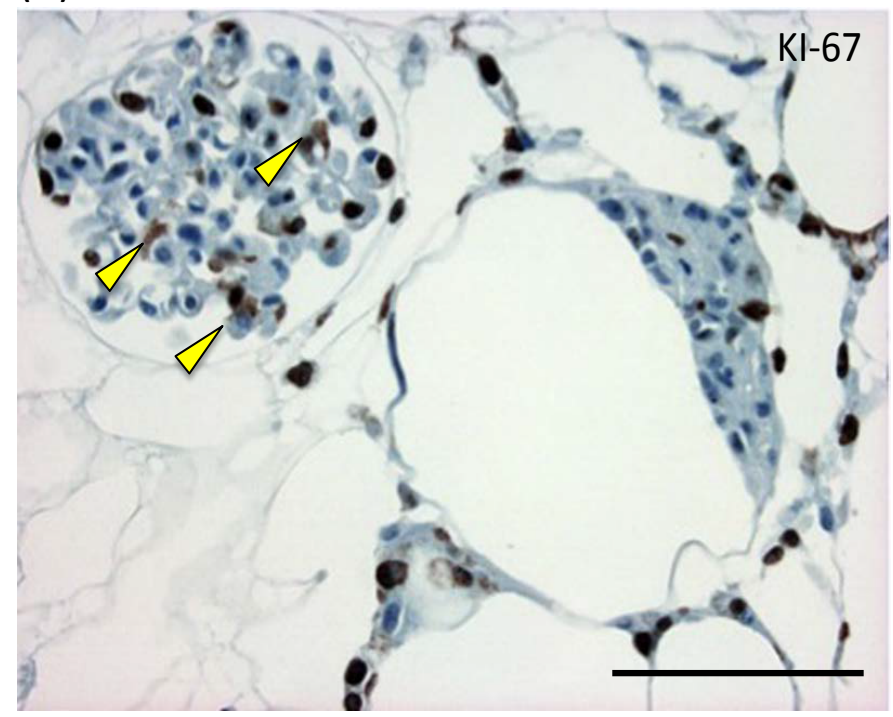

Figure 4. Histological assessment of perfusion-cultured scaffold.

A: HE staining shows a wide distribution of re-endothelialized glomeruli and adjacent vessels. Scale bar, $100 \mu \mathrm{m}$. B: In a densely repopulated glomerulus, KI-67 positive cells (indicated by arrowheads) are observed. Some of endothelial cells dispersed into peritubular spaces beyond the nephron. Scale bar, $100 \mu \mathrm{m}$. and that some of them were present in the peritubular capillaries beyond the glomerulus (Figure 4B).

When $\mathrm{KH}$ solution was perfused at a constant rate into the renal artery of a scaffold that had undergone 72 -hr perfusion culture, the arterial pressure was stable at around $150 \mathrm{mmHg}$. When a non-seeded scaffold was perfused at the same rate, the pressure was approximately half the level in a seeded scaffold, showing that the flow resistance was significantly higher in perfusion-cultured scaffolds (Figure 5A). The results suggest that the engrafted endothelial cells altered the resistance for the perfusate. During perfusion using $100 \mathrm{ml}$ of $\mathrm{KH}$ solution, perfusion-cultured scaffolds produced a tiny amount of effluent in the ureter, whereas non-seeded scaffolds generated a larger volume (Figure $5 B$ ). It seems likely that engrafted cells prevented free filtration inside the scaffold, leading to the reduced volume of effluent in the ureter.

\section{Discussion}

Two routes have commonly been used for repopulating decellularized kidney ECM scaffolds, which are antegrade seeding of the vascular network via the renal artery and retrograde seeding of the parenchyma via the ureter, but the optimal seeding method for each route has not yet been established [15].

Ross et al. [10] manually injected cell suspensions via the renal artery and ureter, and reported considerable distribution of cells in the vasculature but not in the renal parenchyma. They speculated that the renal papilla acted as a barrier to uniform and efficient cell dispersion. Song et al. [9] were the first to report that TPG facilitates cell dispersion in the tubular spaces as well as the vascular network. They repopulated the vasculature and parenchyma simultaneously with TPG.

To further explore the effects of TPG on recellularization, we carried out experiments by applying different levels of TPG for the two repopulation routes and quantified the number of engrafted cells. We determined the strength of TPG for application in an empirical fashion. When TPG was $-200 \mathrm{mmHg}$ or more in the vascular route, the scaffold was likely to be damaged, while $-50 \mathrm{mmHg}$ or more caused barotrauma in the ureteral route. Therefore, we set TPG at -100 and -20 $\mathrm{mmHg}$, respectively, for the vascular and ureteral routes in this study.

We demonstrated that TPG facilitates cell dispersion in the vascular network. While manual injection delivered a considerable number of endothelial cells, more cells were counted after TPG was applied and we observed a significant increase of cell-positive glomeruli. We speculate that TPG delivers cells to more peripheral regions, leading to an increase of cell-positive glomeruli. We also showed that TPG had an impact on retrograde cell dispersion, but the seeding efficiency still seemed to be insufficient for development of parenchymal function. The appropriate strength of TPG seems to vary between different routes and should be determined for a specific route. Even with application of specific TPG, parenchymal recellularization seems to be highly challenging.

To address the challenges related to parenchymal recellularization, Caralt et al. [16] tried a different approach. They infused tubular cells antegradely into the renal artery at $25 \mathrm{ml} / \mathrm{min}(232 \mathrm{mmHg})$, and observed efficient cellular translocation from the vasculature to the parenchyma along with formation of tubular structures. Our findings are in conflict with theirs, since we found that a high pressure is likely to induce barotrauma and a leakage of cells from the scaffold. To explain this discrepancy, we should take into account the fact that decellularization and recellularization protocols vary among studies, including differences of the decellularizing agent and duration of perfusion, the cell seeding methods and cell types used, and bioreactor 


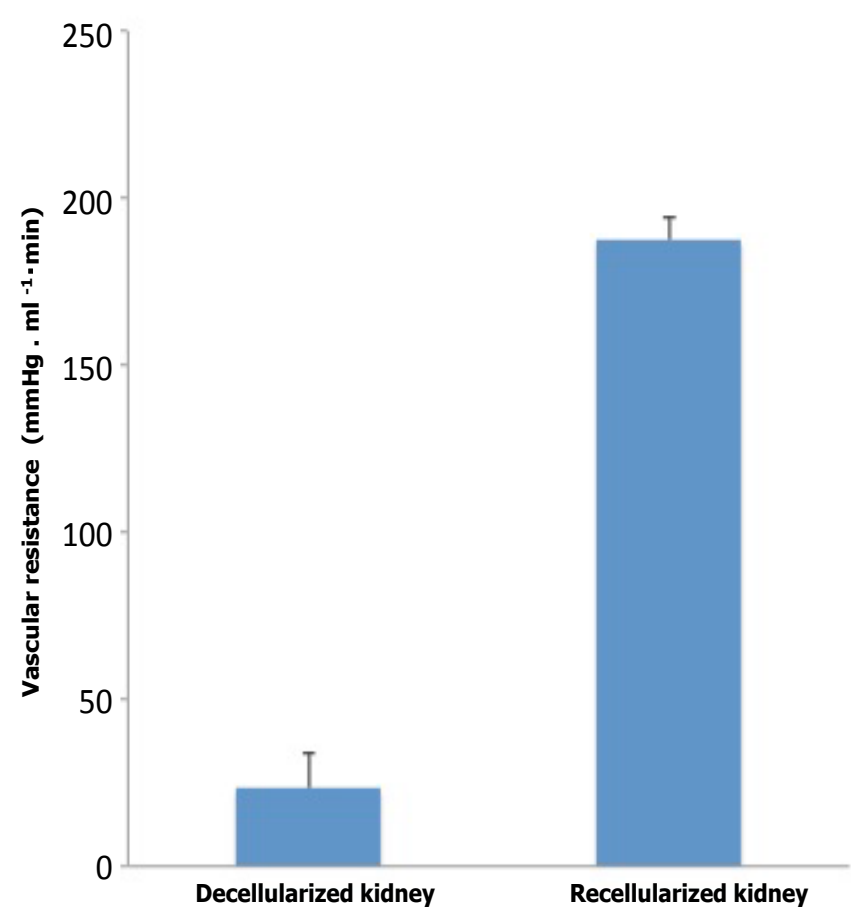

(A)

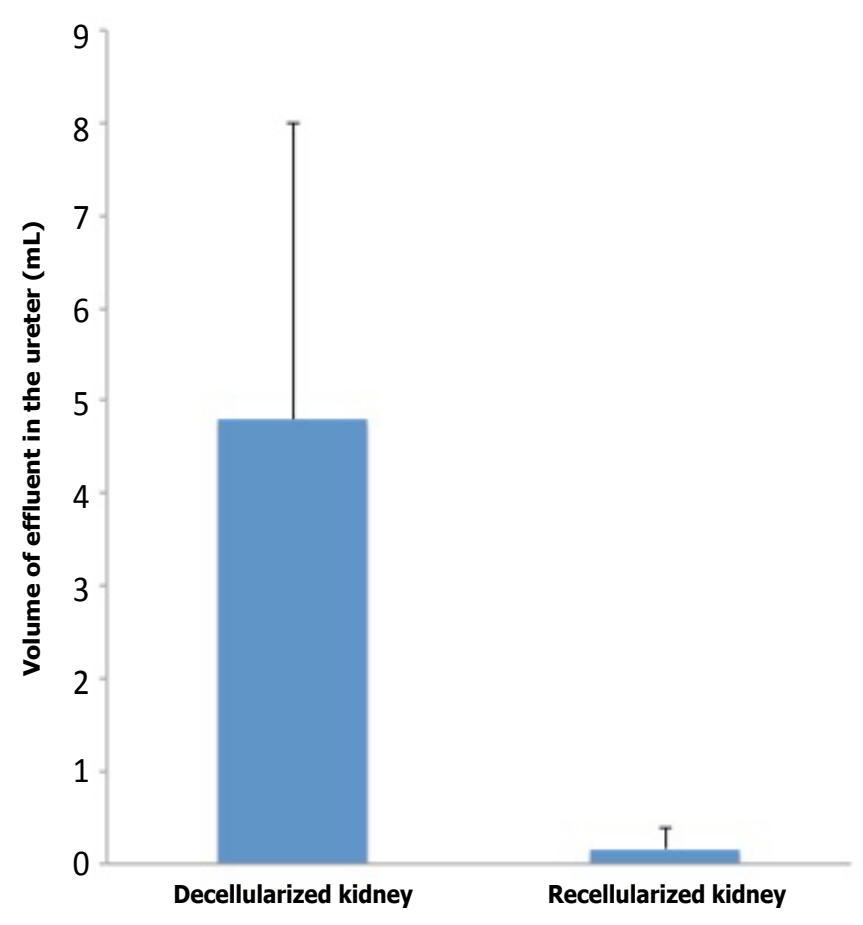

(B)

Figure 5. Functional assessment of perfusion-cultured scaffold. A:

Vascular resistance [arterial pressure $(\mathrm{mmHg}) /$ flow rate $(\mathrm{ml} / \mathrm{min})]$ significantly increased $(p<0.01$, Student's t-test) in re-endothelialized scaffolds $(n=5)$ compared to acellular, nonseeded scaffolds $(n=4)$ when KH solution is arterially delivered. B: Effluent in the ureter significantly decreased $(\mathrm{p}<0.05)$ in re-endotheliarized scaffold $(\mathrm{n}=5)$ compared to acellular, non-seeded scaffold $(\mathrm{n}=4)$ when $\mathrm{KH}$ solution is arterially delivered. parameters that could potentially influence the results [17]. We believe that these variations inevitably place limitations on comparison of recellularization efficiency among studies.

In our functional analysis of scaffolds subjected to perfusion culture, we found that vascular resistance increased after endothelial cell engraftment. This result validated a simulation study by Laurence $e t$ al. [14], which showed that increased cellularity due to recellularization may be associated with decreased porosity and increased pressure in the scaffold. We demonstrated that re-endothelialization increases arterial resistance and reduces the volume of effluent in the ureter, suggesting that these parameters could be used to evaluate recellularization efficiency. As a more specific indicator of renal function, Song et al. [9] analyzed the urinary effluent and determined the capacity for solute clearance of recellularized scaffolds. However, our analysis of effluent was not consistent, showing a wide range among scaffolds. Here also, variation in the type of cell employed (they used highly proliferating cells) and other parameters could lead to differences in the reported results.

In conclusion, our TPG-based seeding method (bolus injection + application of TPG) is safe and effective, since it achieves a stable intra-scaffold pressure and unintended elevation is unlikely. In addition, it does not require a sophisticated and expensive bioreactor with continuous pressure and flow monitoring. Further studies will be required to achieve higher recellularization efficiency, and our seeding method may be useful for research into development of a fully functioning implantable organ.

\section{Acknowledgements}

We are grateful to Ms. C. Sasaki, Institute of Ultrastructural Morphology, St. Marianna University Graduate School of Medicine, for preparing SEM photographs; and to the staff of the Institute for Animal Experimentation, St. Marianna University Graduate School of Medicine, for caring for laboratory rats.

\section{Competing interests}

No competing interests to declare

\section{References}

1. Nakai S, Hanafusa N, Masakane I, Taniguchi M, Hamano T, et al. (2014) An overview of regular dialysis treatment in Japan (as of 31 December 2012). Ther Apher Dial 18: 535-602. [Crossref]

2. Yamagata K, Yagisawa T, Nakai S, Nakayama M, Imai E, et al. (2015) Prevalence and incidence of chronic kidney disease stage G5 in Japan. Clin Exp Nephrol 19: 54-64. [Crossref]

3. Rustad KC, Sorkin M, Levi B, Longaker MT, Gurtner GC (2010) Strategies for organ level tissue engineering. Organogenesis 6: 151-157. [Crossref]

4. Badylak SF, Weiss DJ, Caplan A, Macchiarini P (2012) Engineered whole organs and complex tissues. Lancet 379: 943-952. [Crossref]

5. Soto-Gutierrez A, Wertheim JA, Ott HC, Gilbert TW (2012) Perspectives on wholeorgan assembly: moving toward transplantation on demand. J Clin Invest 122: 38173823. [Crossref]

6. Ott HC, Matthiesen TS, Goh SK, Black LD, Kren SM, et al. (2008) Perfusiondecellularized matrix: using nature's platform to engineer a bioartificial heart. Nat Med 14: 213-221. [Crossref]

7. Uygun BE, Soto-Gutierrez A, Yagi H, Izamis ML, Guzzardi MA, et al. (2010) Organ reengineering through development of a transplantable recellularized liver graft using decellularized liver matrix. Nat Med 16: 814-820. [Crossref]

8. Baptista PM, Siddiqui MM, Lozier G, Rodriguez SR, Atala A, et al. (2011) The use of whole organ decellularization for the generation of a vascularized liver organoid. Hepatology 53: 604-617. [Crossref] 
9. Song JJ, Guyette JP, Gilpin SE, Gonzalez G, Vacanti JP, et al. (2013) Regeneration and experimental orthotopic transplantation of a bioengineered kidney. Nat Med 19: 646-51. [Crossref]

10. Ross EA, Williams MJ, Hamazaki T, Terada N, Clapp WL, et al. (2009) Embryonic stem cells proliferate and differentiate when seeded into kidney scaffolds. J Am Soc Nephrol 20: 2338-2347. [Crossref]

11. Bonandrini B, Figliuzzi M, Papadimou E, Morigi M, Perico N, et al. (2014) Recellularization of well-preserved acellular kidney scaffold using embryonic stem cells. Tissue Eng Part A 20: 1486-1498. [Crossref]

12. Bonenfant NR, Sokocevic D, Wagner DE, Borg ZD, Lathrop MJ, et al. (2013) The effects of storage and sterilization on de-cellularized and re-cellularized whole lung. Biomaterials 34: 3231-3245. [Crossref]

13. Mirmalek-Sani SH, Orlando G, McQuilling JP, Pareta R, Mack DL, et al. (2013) Porcine pancreas extracellular matrix as a platform for endocrine pancreas bioengineering. Biomaterials 34: 5488-5495. [Crossref]

14. Lawrence BJ, Devarapalli M, Madihally SV (2009) Flow dynamics in bioreactor containing tissue engineering scaffolds. Biotechnol Bioeng 102: 935-947. [Crossref]

15. Scarritt ME, Pashos NC2, Bunnell BA3 (2015) A review of cellularization strategies for tissue engineering of whole organs. Front Bioeng Biotechnol 3: 43. [Crossref]

16. Caralt M, Uzarski JS, lacob S, Obergfell KP, Berg N, et al. (2014) Optimization and critical evaluation of decellularization strategies to develop renal extracellular matrix scaffolds as biological templates for organ engineering and transplantation. $\mathrm{Am} \mathrm{J}$ Transplant 15: 64-75. [Crossref]

17. Guyette JP, Gilpin SE, Charest JM, Tapias LF, Ren X, et al. (2014) Perfusion decellularization of whole organs. Nat Protoc 9: 1451-1468. [Crossref]

Copyright: $\odot 2015$ Hachisuka S. This is an open-access article distributed under the terms of the Creative Commons Attribution License, which permits unrestricted use, distribution, and reproduction in any medium, provided the original author and source are credited. 